VERSITA

\author{
TATRA \\ MOUNTaiNS \\ Mathematical Publications \\ DOI: $10.2478 /$ v10127-011-0006-4 \\ Tatra Mt. Math. Publ. 48 (2011), 61-71
}

\title{
OSCILLATION THEOREMS FOR SECOND ORDER ADVANCED NEUTRAL DIFFERENTIAL EQUATIONS
}

\author{
JOZEF DŽURINA
}

\begin{abstract}
The aim of this paper is to study the oscillation of the second order advanced neutral differential equations

$$
\left(r(t)[x(t)+p(t) x(\tau(t))]^{\prime}\right)^{\prime}+q(t) x(\sigma(t))=0 .
$$

Obtained results are based on the new comparison theorems that enable us to reduce problem of the oscillation of the second order equation to the the oscillation of the first order equations. Obtained comparison principles essentially simplify the examination of the studied equations.
\end{abstract}

\section{Introduction}

In this paper, we shall study the oscillation behavior of the solutions of the second order advanced neutral differential equations of the form

$$
\left(r(t)[x(t)+p(t) x(\tau(t))]^{\prime}\right)^{\prime}+q(t) x(\sigma(t))=0,
$$

where $q(t) \in C\left(\left[t_{0}, \infty\right)\right), r(t), p(t), \tau(t), \sigma(t) \in C^{1}\left(\left[t_{0}, \infty\right)\right)$ and

$\left(\mathrm{H}_{1}\right) r(t)>0, q(t)>0,0 \leq p(t) \leq p_{0}<\infty$,

$\left(\mathrm{H}_{2}\right) \lim _{t \rightarrow \infty} \tau(t)=\infty, \sigma(t) \geq t, \sigma(t)$ is nondecreasing,

$\left(\mathrm{H}_{3}\right) \tau^{\prime}(t) \geq \tau_{0}>0, \tau \circ \sigma=\sigma \circ \tau$.

(C) 2011 Mathematical Institute, Slovak Academy of Sciences.

2010 Mathematics Subject Classification: $34 \mathrm{~K} 11,34 \mathrm{C} 10$.

Keywords: second-order neutral differential equations, comparison theorem, oscillation, nonoscillation.

This work was supported by the Slovak Research and Development Agency under the contract No. APVV-0073-07. 


\section{JOZEF DŽURINA}

For our further references, we denote and assume that

$$
R(t)=\int_{t_{0}}^{t} \frac{1}{r(t)} \mathrm{d} s \rightarrow \infty \quad \text { as } \quad t \rightarrow \infty .
$$

We set

$$
z(t)=x(t)+p(t) x(\tau(t)) .
$$

By a solution of equation (E) we mean a function

$$
x(t) \in C\left(\left[T_{x}, \infty\right)\right), \quad T_{x} \geq t_{0},
$$

which has the property $r(t) z^{\prime}(t) \in C^{1}\left(\left[T_{x}, \infty\right)\right)$ and satisfies (E) on $\left[T_{x}, \infty\right)$. A solution $x(t)$ of $(\mathrm{E})$ is said to be proper if

$$
\sup \{|x(t)|: t \geq T\}>0 \quad \text { for all } T \geq T_{x} .
$$

We assume that (E) possesses such a solution. A solution of (E) is called oscillatory if it has arbitrarily large zeros on $\left[T_{x}, \infty\right)$ and otherwise, it is said to be nonoscillatory. Equation (E) itself is said to be oscillatory if all its solutions are oscillatory.

The second order equations have the applications in various problems of physics, biology and economy. Therefore, there is the permanent interest in obtaining sufficient conditions for the oscillation or nonoscillation of the solutions of varietal types of the second order equations.

Since the authors mainly study the oscillation properties of the delay differential equations there is a gap in the oscillation theory. In this paper, we attempt to fill this gap and we shall deal with the advanced neutral differential equations.

Known oscillation criteria require various restrictions on the coefficients of the studied neutral differential equations.

Gram matikopoulos et al. [9] shoved that $0 \leq p(t) \leq 1$ together with

$$
\int_{0}^{\infty} q(s)(1-p(s-\sigma)) \mathrm{d} s=\infty
$$

guarantee the oscillation of the neutral equation

$$
(x(t)+p(t) x(t-\tau))^{\prime \prime}+q(t) x(t-\sigma)=0 .
$$

For the same equation Erbe et al. [7] established oscillation criterion that requires

$$
q(t) \geq q_{0}>0, \quad p_{1} \leq p(t) \leq p_{2}, \quad p(t) \text { not eventually negative. }
$$

This result has been improved and generalized by other authors. We mention Grace and Lalli [8] who studied oscillation of

$$
\left(r(t)[x(t)+p(t) x(t-\tau)]^{\prime}\right)^{\prime}+q(t) f(x(t-\sigma))=0,
$$


under the conditions

$$
\frac{f(x)}{x} \geq k, \quad \int_{0}^{\infty} \frac{\mathrm{d} s}{r(s)}=\infty
$$

and

$$
\int_{0}^{\infty} \rho(s) q(s)(1-p(s-\sigma))-\frac{\left(\rho^{\prime}(s)\right)^{2} r(s-\sigma)}{4 k \rho(s)} \mathrm{d} s=\infty,
$$

where $\rho(t)$ is an optional function.

$\mathrm{X} \mathrm{u}$ and $\mathrm{X}$ ia [17 established oscillation of

$$
(x(t)+p(t) x(t-\tau))^{\prime \prime}+q(t) f(x(t-\sigma))=0,
$$

provided that

$$
0 \leq p(t)<\infty, \quad q(t) \geq M>0 .
$$

$\mathrm{Li}$ at al. [12] studied the more general neutral differential equation

$$
\left(r(t)[x(t)+p(t) x(\tau(t))]^{\prime}\right)^{\prime}+q(t) f(x(\sigma(t)))=0 .
$$

Using double Riccati transformation, they presented new oscillation criteria, where they replaced usually used restriction $0 \leq p(t) \leq 1$ with $0 \leq p(t) \leq p_{0}<$ $\infty$, and also required inter alia $\sigma(t) \leq \tau(t) \leq t$, and

$$
\int_{0}^{\infty} \rho(s) q(s)(1-p(s-\sigma))-\frac{\left(\rho^{\prime}(s)\right)^{2} r(s-\sigma)}{4 k \rho(s)} \mathrm{d} s=\infty,
$$

where $\rho(t)$ is an optional function.

In this paper, we use a different technique for studying the oscillation of (E). We shall establish new comparison theorems in which we compare the second order equation (E) with the first order advanced differential inequality in the sense that the absence of the positive solutions of this first order inequality yields the oscillation of (E). Established comparison theorems essentially simplify the examination of $(\mathrm{E})$ and enable us also to eliminate some conditions imposed in the cited papers on the coefficients of (E). Moreover, our results can be easily extended to cover also the more general functional differential equations.

Remark 1. All functional inequalities considered in this paper are assumed to hold eventually, that is they are satisfied for all $t$ large enough.

Remark 2. Without loss of generality, we can deal only with positive solutions of (E). 
JOZEF DŽURINA

\section{Main results}

It follows from (1.1) that the positive solutions of (E) have the following property.

LEMMA 1. If $x(t)$ is a positive solution of (E), then the corresponding function

$$
z(t)=x(t)+p(t) x(\tau(t))
$$

satisfies

$$
z(t)>0, \quad r(t) z^{\prime}(t)>0, \quad\left(r(t) z^{\prime}(t)\right)^{\prime}<0
$$

eventually.

P r o of. Assume that $x(t)$ is a positive solution of (E). Then it follows from (E) that

$$
\left(r(t) z^{\prime}(t)\right)^{\prime}=-q(t) x(\sigma(t))<0 .
$$

Consequently, $r(t) z^{\prime}(t)$ is decreasing and thus either $z^{\prime}(t)>0$ or $z^{\prime}(t)<0$, eventually. If we let $z^{\prime}(t)<0$, then also $r(t) z^{\prime}(t)<-c<0$ and integrating this from $t_{1}$ to $t$, we have

$$
z(t) \leq z\left(t_{1}\right)-c \int_{t_{1}}^{\infty} \frac{1}{r(s)} \mathrm{d} s \rightarrow-\infty \quad \text { as } \quad t \rightarrow \infty .
$$

This contradicts the positivity of $z(t)$ and the proof is complete.

For our further references, let us denote

$$
\begin{aligned}
Q(t) & =\min \{q(t), q(\tau(t))\}, \quad Q_{1}(t)=\frac{1}{r(t)} \int_{t}^{\infty} Q(s) \mathrm{d} s, \\
Q_{2}(t) & =Q(t)\left(R(t)-R\left(t_{1}\right)\right),
\end{aligned}
$$

where $t \geq t_{1}, t_{1}$ is large enough.

TheOREm 1. Let $\tau(t) \geq t$. Assume that at least one of the first order advanced differential inequalities

$$
\begin{aligned}
& w^{\prime}(t)-\frac{\tau_{0}}{\tau_{0}+p_{0}} Q_{1}(t) w(\sigma(t)) \geq 0, \\
& w^{\prime}(t)-\frac{\tau_{0}}{\tau_{0}+p_{0}} Q_{2}(t) w(\sigma(t)) \geq 0,
\end{aligned}
$$

has no positive solution. Then (E) is oscillatory. 


\section{OSCILLATION THEOREMS}

P r o of. Assume that $x(t)$ is a positive solution of $(\mathrm{E})$. Then the corresponding function $z(t)$ satisfies

$$
\begin{aligned}
z(\sigma(t)) & =x(\sigma(t))+p(\sigma(t)) x(\tau(\sigma(t))) \\
& \leq x(\sigma(t))+p_{0} x(\sigma(\tau(t)))
\end{aligned}
$$

where we have used the hypothesis $\left(\mathrm{H}_{3}\right)$.

On the other hand, it follows from (E) that

$$
\left(r(t) z^{\prime}(t)\right)^{\prime}+q(t) x(\sigma(t))=0,
$$

and moreover, taking $\left(\mathrm{H}_{1}\right)$ and $\left(\mathrm{H}_{3}\right)$ into account, we have

$$
\begin{aligned}
0 & =\frac{1}{\tau^{\prime}(t)}\left(r(\tau(t)) z^{\prime}(\tau(t))\right)^{\prime}+q(\tau(t)) x(\sigma(\tau(t))) \\
& \geq \frac{p_{0}}{\tau_{0}}\left(r(\tau(t)) z^{\prime}(\tau(t))\right)^{\prime}+p_{0} q(\tau(t)) x(\sigma(\tau(t))) .
\end{aligned}
$$

Combining (2.5) and (2.6), we are led to

$$
\left(r(t) z^{\prime}(t)\right)^{\prime}+\frac{p_{0}}{\tau_{0}}\left(r(\tau(t)) z^{\prime}(\tau(t))\right)^{\prime}+q(t) x(\sigma(t))+p_{0} q(\tau(t)) x(\sigma(\tau(t))) \leq 0,
$$

which in view of (2.4) and (2.2) provides

$$
\left(r(t) z^{\prime}(t)\right)^{\prime}+\frac{p_{0}}{\tau_{0}}\left(r(\tau(t)) z^{\prime}(\tau(t))\right)^{\prime}+Q(t) z(\sigma(t)) \leq 0 .
$$

Integrating the previous inequality from $t$ to $\infty$, we get

$$
r(t) z^{\prime}(t)+\frac{p_{0}}{\tau_{0}} r(\tau(t)) z^{\prime}(\tau(t)) \geq \int_{t}^{\infty} Q(s) z(\sigma(s)) \mathrm{d} s .
$$

On the other hand, since $r(t) z^{\prime}(t)$ is decreasing and $\tau(t) \geq t$, it follows from (2.8) that

$$
r(t) z^{\prime}(t)\left(1+\frac{p_{0}}{\tau_{0}}\right) \geq \int_{t}^{\infty} Q(s) z(\sigma(s)) \mathrm{d} s .
$$

Using that $z(\sigma(t))$ is increasing, an integration from $t_{1}$ to $t$, yields

$$
\begin{aligned}
z(t) & \geq \frac{\tau_{0}}{p_{0}+\tau_{0}} \int_{t_{1}}^{t} \frac{1}{r(u)} \int_{u}^{\infty} Q(s) z(\sigma(s)) \mathrm{d} s \mathrm{~d} u \\
& \geq \frac{\tau_{0}}{p_{0}+\tau_{0}} \int_{t_{1}}^{t} z(\sigma(u)) \frac{1}{r(u)} \int_{u}^{\infty} Q(s) \mathrm{d} s \mathrm{~d} u .
\end{aligned}
$$


That is,

$$
z(t) \geq \frac{\tau_{0}}{p_{0}+\tau_{0}} \int_{t_{1}}^{t} Q_{1}(u) z(\sigma(u)) \mathrm{d} u .
$$

Let us denote the right hand side of (2.10) by $w(t)$. Then $w(t)>0$ and using $z(t) \geq w(t)$, one can see that

$$
w^{\prime}(t)=\frac{\tau_{0}}{p_{0}+\tau_{0}} Q_{1}(t) z(\sigma(t)) \geq \frac{\tau_{0}}{p_{0}+\tau_{0}} Q_{1}(t) w(\sigma(t)) .
$$

Thus $w(t)$ is a positive solution of $\left(\mathrm{E}_{2}\right)$. This contradicts our assumptions and thus the absence of the positive solutions of $\left(\mathrm{E}_{2}\right)$ implies the oscillation of $(\mathrm{E})$.

Now, we shall show that the absence of the positive solutions of $\left(\mathrm{E}_{2}\right)$ also yields the oscillation of (E). An integration of (2.9) from $t_{1}$ to $t$, provides

$$
\begin{aligned}
z(t) & \geq \frac{\tau_{0}}{p_{0}+\tau_{0}} \int_{t_{1}}^{t} \frac{1}{r(u)} \int_{u}^{\infty} Q(s) z(\sigma(s)) \mathrm{d} s \mathrm{~d} u \\
& \geq \frac{\tau_{0}}{p_{0}+\tau_{0}} \int_{t_{1}}^{t} \frac{1}{r(u)} \int_{u}^{t} Q(s) z(\sigma(s)) \mathrm{d} s \mathrm{~d} u \\
& =\frac{\tau_{0}}{p_{0}+\tau_{0}} \int_{t_{1}}^{t} Q(s) z(\sigma(s)) \int_{t_{1}}^{s} \frac{1}{r(u)} \mathrm{d} u \mathrm{~d} s .
\end{aligned}
$$

That is,

$$
z(t) \geq \frac{\tau_{0}}{p_{0}+\tau_{0}} \int_{t_{1}}^{t} Q_{2}(s) z(\sigma(s)) \mathrm{d} s .
$$

Let us denote the right hand side of (2.11) by $w(t)$. Then $w(t)>0$ and using that $z(t) \geq w(t)$, one can see that $w(t)$ is a positive solution of $\left(\mathrm{E}_{3}\right)$. This is a contradiction and the proof is complete now.

Corollary 1. Let $\tau(t) \geq t$. Assume that at least one of the following conditions

$$
\begin{aligned}
& \liminf _{t \rightarrow \infty} \int_{t}^{\sigma(t)} Q_{1}(s) \mathrm{d} s>\frac{\tau_{0}+p_{0}}{\tau_{0} \mathrm{e}}, \\
& \liminf _{t \rightarrow \infty} \int_{t}^{\sigma(t)} Q_{2}(s) \mathrm{d} s>\frac{\tau_{0}+p_{0}}{\tau_{0} \mathrm{e}},
\end{aligned}
$$

holds. Then (E) is oscillatory. 


\section{OSCILLATION THEOREMS}

P r o o f. Theorem 2.1.1 from [10] guarantees that ( $\left.\mathrm{E}_{1}\right)$ and $\left(\mathrm{E}_{2}\right)$ have no positive solutions provided that (2.12) and (2.13) hold, respectively. The assertion now follows from Theorem 1 .

For our incoming references, let us denote

$$
Q_{3}(t)=\frac{\tau^{\prime}(t)}{r(\tau(t))} \int_{t}^{\infty} Q(s) \mathrm{d} s, \quad Q_{4}(t)=Q(t)\left(R(\tau(t))-R\left(t_{1}\right)\right) .
$$

where $t \geq t_{1}, t_{1}$ is large enough.

Theorem 2. Let $\tau(t) \leq t$. Assume that at least one of the first order advanced differential inequalities

$$
\begin{aligned}
& w^{\prime}(t)-\frac{\tau_{0}}{\tau_{0}+p_{0}} Q_{3}(t) w\left(\tau^{-1}(\sigma(t))\right) \geq 0, \\
& w^{\prime}(t)-\frac{\tau_{0}}{\tau_{0}+p_{0}} Q_{4}(t) w\left(\tau^{-1}(\sigma(t))\right) \geq 0,
\end{aligned}
$$

has no positive solution. Then (E) is oscillatory.

P r o of. Assume that $x(t)$ is a positive solution of $(\mathrm{E})$. Then the corresponding function $z(t)$ satisfies (2.8). On the other hand, since $r(t) z^{\prime}(t)$ is decreasing and $\tau(t) \leq t$, then it follows from (2.8) that

$$
r(\tau(t)) z^{\prime}(\tau(t))\left(1+\frac{p_{0}}{\tau_{0}}\right) \geq \int_{t}^{\infty} Q(s) z(\sigma(s)) \mathrm{d} s .
$$

Multiplying by $\tau^{\prime}(t) / r(\tau(t))$ and then integrating from $t_{1}$ to $t$, we get

$$
\begin{aligned}
z(\tau(t)) & \geq \frac{\tau_{0}}{p_{0}+\tau_{0}} \int_{t_{1}}^{t} \frac{\tau^{\prime}(u)}{r(\tau(u))} \int_{u}^{\infty} Q(s) z(\sigma(s)) \mathrm{d} s \mathrm{~d} u \\
& \geq \frac{\tau_{0}}{p_{0}+\tau_{0}} \int_{t_{1}}^{t} z(\sigma(u)) \frac{\tau^{\prime}(u)}{r(\tau(u))} \int_{u}^{\infty} Q(s) \mathrm{d} s \mathrm{~d} u .
\end{aligned}
$$

That is,

$$
z(\tau(t)) \geq \frac{\tau_{0}}{p_{0}+\tau_{0}} \int_{t_{1}}^{t} Q_{3}(u) z(\sigma(u)) \mathrm{d} u .
$$

Let us denote the right hand side of (2.16) by $w(t)$. Noting that

$$
z(\tau(t)) \geq w(t)
$$


one can see that $w(t)$ is a positive solution of $\left(\mathrm{E}_{3}\right)$. This contradicts our assumptions and thus the absence of the positive solutions of $\left(\mathrm{E}_{3}\right)$ implies the oscillation of (E).

Now, we shall show that the absence of the positive solutions of $\left(\mathrm{E}_{4}\right)$ implies the oscillation of (E). An integration of (2.15) from $t_{1}$ to $t$, gives

$$
\begin{aligned}
z(\tau(t)) & \geq \frac{\tau_{0}}{p_{0}+\tau_{0}} \int_{t_{1}}^{t} \frac{\tau^{\prime}(u)}{r(\tau(u))} \int_{u}^{\infty} Q(s) z(\sigma(s)) \mathrm{d} s \mathrm{~d} u \\
& \geq \frac{\tau_{0}}{p_{0}+\tau_{0}} \int_{t_{1}}^{t} \frac{\tau^{\prime}(u)}{r(\tau(u))} \int_{u}^{t} Q(s) z(\sigma(s)) \mathrm{d} s \mathrm{~d} u \\
& =\frac{\tau_{0}}{p_{0}+\tau_{0}} \int_{t_{1}}^{t} Q(s) z(\sigma(s)) \int_{t_{1}}^{s} \frac{\tau^{\prime}(u)}{r(\tau(u))} \mathrm{d} u \mathrm{~d} s .
\end{aligned}
$$

That is,

$$
z(\tau(t)) \geq \frac{\tau_{0}}{p_{0}+\tau_{0}} \int_{t_{1}}^{t} Q_{4}(s) z(\sigma(s)) \mathrm{d} s .
$$

Let us denote the right hand side of (2.17) by $w(t)$. Then $w(t)>0$ and using that $z(\tau(t)) \geq w(t)$, one can see that $w(t)$ is a positive solution of $\left(\mathrm{E}_{4}\right)$. This is a contradiction and the proof is complete now.

Corollary 2. Let $\tau(t) \leq t$. Assume that at least one of the following conditions

$$
\begin{aligned}
\liminf _{t \rightarrow \infty} & \int_{t}^{\tau^{-1}(\sigma(t))} Q_{3}(s) \mathrm{d} s>\frac{\tau_{0}+p_{0}}{\tau_{0} \mathrm{e}}, \\
\liminf _{t \rightarrow \infty} & \int_{t}^{\tau^{-1}(\sigma(t))} Q_{4}(s) \mathrm{d} s>\frac{\tau_{0}+p_{0}}{\tau_{0} \mathrm{e}},
\end{aligned}
$$

holds. Then (E) is oscillatory.

P r o o f. Theorem 2.1.1 from [10] guarantees that ( $\left.\mathrm{E}_{3}\right)$ and $\left(\mathrm{E}_{4}\right)$ have no positive solutions provided that (2.18) and (2.19) hold, respectively. The assertion now follows from Theorem 2 ,

Remark 3. The condition $\tau \circ \sigma=\sigma \circ \tau$ contained in the hypothesis $\left(\mathrm{H}_{3}\right)$ is satisfies if the deviating arguments $\tau(t)$ and $\sigma(t)$ are of the same form, that is, e.g., $\tau(t)=\alpha t$ and at the same time $\sigma(t)=\beta t$ or $\tau(t)=t^{\alpha}$ and simultaneously $\sigma(t)=t^{\beta}$, etc. 


\section{OSCILLATION THEOREMS}

EXAMPLE 1. We consider the second order neutral differential equation

$$
\left(t^{1 / 2}\left[x(t)+p_{0} x\left(\tau_{0} t\right)\right]^{\prime}\right)^{\prime}+\frac{a}{t^{3 / 2}} x(\alpha t)=0,
$$

where $0 \leq p_{0}<\infty, 0<\tau_{0}<\infty, \alpha>1$, and $a>0$.

If $\tau_{0} \geq 1$, then

$$
Q(t)=q(\tau(t))=\frac{a}{\left(\tau_{0} t\right)^{\frac{3}{2}}}
$$

and moreover, the conditions (2.12) and (2.13) of Corollary 1 reduce to

$$
a \ln \alpha>\frac{\left(\tau_{0}+p_{0}\right) \sqrt{\tau_{0}}}{2 \tau_{0} \mathrm{e}},
$$

which guaranties oscillation of $\left(\mathrm{E}_{5}\right)$.

If $\tau_{0} \leq 1$, then $Q(t)=q(t)=a / t^{\frac{3}{2}}$ and conditions (2.18) and (2.19) of Corollary 2 reduce to

$$
a \ln \frac{\alpha}{\tau_{0}}>\frac{\tau_{0}+p_{0}}{2 \tau_{0}^{\frac{3}{2}} \mathrm{e}}
$$

which also guaranties oscillation of $\left(\mathrm{E}_{5}\right)$. Consequently, we have covered oscillation of $\left(\mathrm{E}_{5}\right)$ for all $\alpha \in(0, \infty)$, that is for $\tau(t)=\alpha t$ to be delay or advanced argument.

On the other hand, if

$$
a=\left(\frac{1}{2}-\gamma\right) \gamma\left(1+p_{0} \tau_{0}^{\gamma}\right) \alpha^{-\gamma}, \quad \text { with } \quad \gamma \in\left(0, \frac{1}{2}\right),
$$

then both presented oscillation criteria fail and $\left(\mathrm{E}_{5}\right)$ has a nonoscillatory solution $x(t)=t^{\gamma}$.

Remark 4. All our conclusions can be very easily extend to nonlinear neutral differential

$$
\left(r(t)[x(t)+p(t) x(\tau(t))]^{\prime}\right)^{\prime}+q(t) f(x(\sigma(t)))=0 .
$$

Adding the additional condition

$$
\frac{f(x)}{x} \geq k
$$

the reader can verify that our results here hold also for $\left(\mathrm{E}_{5}\right)$, provided that we replace in the assumption of our achievements the function $q(t)$ by $k q(t)$. 


\section{JOZEF DŽURINA}

\section{Summary}

In this paper we have introduced new comparison theorems for investigation of the oscillation of $(\mathrm{E})$. The established comparison principles reduce oscillation of the second order advanced neutral equations to studying properties of various types of the first order advanced differential inequalities, which essentially simplifies examination of (E). Our technique permits to relax restrictions usually imposed on the coefficients of $(\mathrm{E})$. So that our results are of high generality and can be easily extended also to the nonlinear neutral differential equations. Obtained results are easily applicable and are illustrated on a suitable example.

\section{REFERENCES}

[1] AGARWAL, R. P.-GRACE, S. R.: Oscillation theorems for certain neutral functional differential equations, Comput. Math. Appl. 38 (1999), 1-11.

[2] BACULÍKOVÁ, B.: Oscillation criteria for second order nonlinear differential equations, Arch. Math. (Brno) 42 (2006), 141-149.

[3] BACULÍKOVÁ, B.: Oscillation theorems for third order neutral differential equations, Tatra Mt. Math. Publ., 2011 (to apear).

[4] BACULÍKOVÁ, B.-LACKOVÁ, D.: Oscillation criteria for second order retarded differential equations, Stud. Univ. Žilina, Math. Ser. 20 (2006), 11-18.

[5] BAINOV, D. D.-MISHEV, D. P.: Oscillation Theory for Nonlinear Differential Equations with Delay. Adam Hilger, Bristol, 1991.

[6] DŽURINA, J.-STAVROULAKIS, I. P.: Oscillation criteria for second order delay differential equations, Appl. Math. Comput. 140 (2003), 445-453.

[7] ERBE, L. H.-KONG, Q.-ZHANG, B. G.: Oscillation Theory for Functional Differential Equations. Marcel Dekker, New York, 1994.

[8] GRACE, S. R.-LALLI, B. S.: Oscillation of nonlinear second order neutral delay differential equations, Rad. Math. 3 (1987), 77-84.

[9] GRAMMATIKOPOULOS, M. K.-LADAS, G.-MEIMARIDOU, A.: Oscillation of second order neutral delay differential equation, Rad. Math. 1 (1985), 267-274.

[10] LADDE, G. S.-LAKSHMIKANTHAM, V.-ZHANG, B. G.: Oscillation Theory of Differential Equations with Deviating Arguments. Marcel Dekker, New York, 1987.

[11] KIgURADZE, I. T.-CHATURIA, T. A.: Asymptotic Properties of Solutions of Nonautonomous Ordinary Differential Equations. Kluwer Acad. Publ., Dordrecht, 1993.

[12] LI, T.-HAN, Z.-ZHANG, CH.-SUN, S.: Oscillation theorems for second-order neutral functional differential equations, J. Appl. Anal. (to appear).

[13] LIN, X.-TANG, X. H.: Oscillation of solutions of neutral differential equations with superlinear neutral term, Appl. Math. Lett. 20 (2007), 1016-1022.

[14] LIU, L. H.-BAI, Z.: New oscillation criteria for second-order nonlinear neutral delay differential equations, J. Comput. Appl. Math. 231 (2009), 657-663.

[15] HASANBULLI, M.-ROGOVCHENKO, Y.: Oscillation criteria for second order nonlinear neutral differential equations, Appl. Math. Comp. 215 (2010), 4392-4399. 


\section{OSCILLATION THEOREMS}

[16] ROGOVCHENKO, Y.-TUNCAY, F.: Oscillation criteria for second order nonlinear differential equations with damping, Nonlinear Anal. 69 (2008), 208-221.

[17] XU, R.-XIA, Y.: A note on the oscillation of second-order nonlinear neutral functional differential equations, J. Contemp. Math. Sci. 3 (2008), 1441-1450.

[18] XU, R.-MENG, F.: Some new oscillation criteria for second order quasi-linear neutral delay differential equations, Appl. Math. Comput. 182 (2006), 797-803.

[19] XU, R.-MENG, F.: Oscillation criteria for second order quasi-linear neutral delay differential equations, Appl. Math. Comput. 192 (2007), 216-222.

Received September 29, 2010

Department of Mathematics

Faculty of Electrical Engineering and

Informatics

Technical University of Košice

Letná 9

SK-042-00 Košice

SLOVAKIA

E-mail: jozef.dzurina@tuke.sk 